\author{
R. BENNEWITZ ,* \\ S. SCHÄR \\ E. GNECCO \\ O. PFEIFFER \\ M. BAMMERLIN \\ E. MEYER
}

\section{Atomic structure of alkali halide surfaces}

\author{
Institute of Physics, University of Basel, 4056 Basel, Switzerland
}

\section{Received: 28 March 2003/Accepted: 21 October 2003 Published online: 3 February 2004 • (C) Springer-Verlag 2004}

ABSTRACT The atomic structure of surfaces of alkali halide crystals has been revealed by means of high-resolution dynamic force microscopy. True atomic resolution is demonstrated both on steps surrounding islands or pits, and on a chemically mixed crystal. We have directly observed the enhanced interaction at low-coordinated sites by force microscopy. The growth of $\mathrm{NaCl}$ films on metal surfaces and radiation damage in a $\mathrm{KBr}$ surface is discussed based on force microscopy results. The damping of the tip oscillation in dynamic force microscopy might provide insight into dissipation processes on the atomic scale. Finally, we present atomically resolved images of wear debris found after scratching a $\mathrm{KBr}$ surface.

PACS 68.37.-d; 68.37.Ps; 75.55.Fv

\section{1}

\section{Introduction}

Dynamic force microscopy has been developed in recent years into a method capable of atomic resolution microscopy on different types of surfaces, including semiconductors, metals, or oxides [1]. A class of insulating materials where particularly clear results have been obtained are alkali halide crystals. The comparably simple preparation of clean surfaces and the strong interaction contrast of the ionic bonds on short length scale contributed to the experimental progress [2].

In dynamic force microscopy, a tip attached to the end of a micro-fabricated cantilever oscillates with an amplitude of $2-10 \mathrm{~nm}$ at the mechanical resonance frequency of the cantilever, typically between $70 \mathrm{kHz}$ and $350 \mathrm{kHz}$. Forces between the tip and the sample influence the oscillation: The increasing attraction between the tip and the sample decreases the resonance frequency. This frequency shift can be used as a feedback signal to control the tip-sample distance. Scanning the tip at constant frequency shift provides an image closely related to the topography of the surface. More accurately, a surface resembling a constant geometric mean of force and interaction energy is recorded [3], as long as the oscillation

E-mail: roland.bennewitz@mcgill.ca

* Now at: McGill University, Montreal, Canada amplitude is far larger than the range of forces. The difference between such measurements and the ideal atomic topography becomes evident when a local variation of forces is encountered, as we will discuss in the following paragraphs [4].

The surface structure of alkali halide crystals has been studied for several decades, starting with the classical replica imaging of step decoration by Bassett [5] or Bethge [6]. Steps emerging from dislocations after heating in a vacuum were imaged in order to study both the nature of dislocations and the evaporation process along monatomic steps. The surface of alkali halide crystals was also in the focus of studies regarding the effects of electronic excitation, in particular when desorption induced by electronic transitions was involved. Recently, a review of this field has been given by Itoh and Stoneham [7]. In this contribution, we intend to describe some recent force microcopy results which may contribute to the understanding of processes at alkali halide surfaces through its ability of atomic resolution imaging. While the paragraphs on film growth and radiation damage include experiments complementary to results by other methods like energy diffraction or desorption spectroscopy, the paragraphs on local dissipation measurements and on wear studies on the nanometer scale are based on results unique for highresolution force microscopy.

\section{$2 \quad$ NaCl films on metal surfaces}

Thin films of $\mathrm{NaCl}$ can be easily grown on flat metal and semiconductor surfaces by molecular evaporation from powder in a crucible. Electron diffraction and scanning tunneling microscopy experiments have revealed that monatomic steps on a $\mathrm{Ge}(100)$ substrate are overgrown by a continuous $\mathrm{NaCl}$ film in a carpet-like mode, due to the internal cohesion of the film being much stronger than the interaction with the substrate $[8,9]$. This growth mode has been confirmed for the $\mathrm{Cu}(111)$ surface by dynamic force microscopy experiments. $\mathrm{NaCl}$ islands extending over several hundred nanometers were found to smoothly cover several substrate steps [10]. In contrast, $\mathrm{NaCl}$ evaporated on $\mathrm{Al}(111)$ has been reported to grow in small islands nucleated at substrate steps [11]. A dynamic force microscopy image of this surface is given in Fig. 1a. For low coverage, islands of $10-20 \mathrm{~nm}$ lateral size start to grow not only at steps but also on terraces. The perfectly 


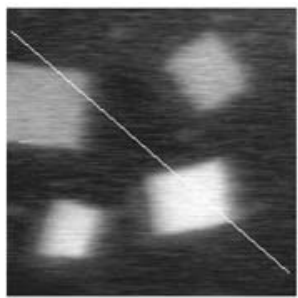

FIGURE 1 Sub-monolayer $\mathrm{NaCl}$ film grown on a $\mathrm{Al}(111)$ surface at room temperature. a Topography image acquired by dynamic force microscopy (frame size $100 \mathrm{~nm}$ ). b Cross-section along the direction indicated by the white line. Note that both monolayer and double-layer islands have grown (amplitude $A=0.7 \mathrm{~nm}$, resonance frequency $f_{0}=165784 \mathrm{~Hz}$, $U_{\text {bias }}=0.32 \mathrm{~V}$, frequency shift $\Delta f=-60 \mathrm{~Hz}$ )

rectangular shape of the islands indicates the strong tendency to minimize the number of corner sites and the mobility of the $\mathrm{NaCl}$ molecules on the surface at room temperature.

From scanning tunneling microscopy the thickness of the $\mathrm{NaCl}$ islands is difficult to judge. Constant current images correspond roughly to surfaces of constant density of states at the Fermi edge. The thickness of insulating layers will appear systematically smaller than geometrically expected, since they provide no electronic states themselves but can only extend states of the substrate $[9,11]$. In dynamic force microscopy there exists a similar problem. The force at a given distance between tip and $\mathrm{Al}$ substrate on the one hand and $\mathrm{NaCl}$ islands on the other hand can differ significantly. Such a difference is largely caused by a variation of the electrostatic force contribution. The substrate and the islands have work functions as different as $1 \mathrm{~V}$ [12]. However, for the experiment represented in Fig. 1 the sample bias has been adjusted such that the same electrostatic contribution to the total force is encountered on substrate and islands. By this means, the geometric island height is well reproduced, and we find that both monolayer and double-layer islands grow directly on the $\mathrm{Al}(111)$ surface. The existence of monolayer islands excludes a growth mode exclusively based on $\mathrm{NaCl}$ molecules in vertical configuration, as suggested by the growth on $\mathrm{Ge}(100)$ where only double-layer islands were found [9].

A high-resolution image of a $\mathrm{NaCl}$ film grown on $\mathrm{Cu}(111)$ is presented in Fig. 2a. Atomic resolution is obtained on both the $\mathrm{NaCl}$ film and a small $\mathrm{NaCl}$ island grown on top of it. The corresponding atomic structure of the island is schematically depicted in Fig. 2b. Note that only one type of ion has been imaged as a protrusion. This is always the case for dynamic force and scanning tunneling microscopy alkali halide surfaces $[2,9,11,13]$. For an interpretation of the atomic contrast in scanning tunneling microscopy, calculations of the local density of states have been performed [11]. They indicate that the highest density of states around the Fermi edge coincides with the sites of chlorine ions, which show up as protrusions. For dynamic force microscopy, the contrast on the atomic scale depends on the atomic structure of the tip apex. Assuming that the charge of an ionic species at the tip apex determines repulsion or attraction at a given surface ion, the actual contrast may even change when the tip touches the surface and picks up ions [14].
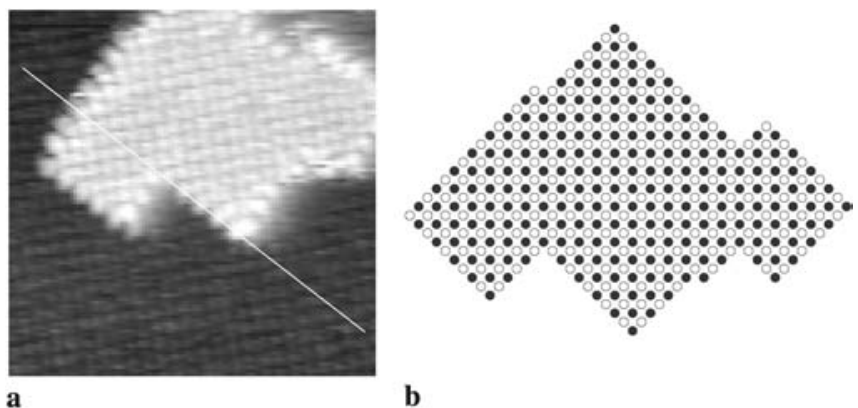

a

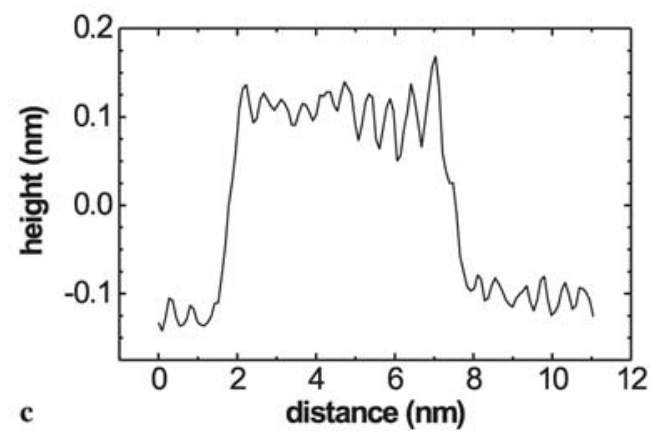

FIGURE $2 \mathrm{NaCl}$ island on top of a $\mathrm{NaCl}$ film grown on $\mathrm{Cu}(111)$. a Dynamic force microscopy image showing atomic resolution on island and film. Note the enhanced corrugation at the steps (amplitude $A=4.6 \mathrm{~nm}$, resonance frequency $f_{0}=156330 \mathrm{~Hz}$, spring constant $k=24 \mathrm{~N} / \mathrm{m}$, frequency shift $\left.\Delta f=-224 \mathrm{~Hz}, U_{\text {bias }}=0.2 \mathrm{~V}\right)$. b Corresponding sketch of the atomic structure of the island. c Cross-section along the white line (from [2])

The cross section in Fig. 2c quantifies the corrugation of the measured topography. There is a significant increase of the corrugation from the terraces over steps to corner sites. This increased corrugation reflects the specific role of lowcoordinated atoms at the surface. The enhanced interaction at low-coordinated sites has many impacts like catalytic activity or nucleation of clusters after evaporation of materials onto the surface. The latter has been used for the imaging of steps using replica techniques, and is still of interest for the study of clusters on non-interacting substrates (see for example a recent study of the growth of magnetic clusters on $\mathrm{NaCl}$ [15]). Pushing the resolution of dynamic force microscopy to the atomic scale provides real-space insights of such enhanced interactions. A detailed analysis of the experiment by means of atomistic simulations has revealed that not only the stronger electrostatic forces around the exposed ions but also their stronger displacement in the force field of the tip contribute to the corrugation [14]. The maximal corrugation found on several alkali halide surfaces is around $0.1 \mathrm{~nm}$. Further approach of the tip results in jumps of atoms and destruction of tip or surface. Such corrugation of $0.1 \mathrm{~nm}$ is far beyond the difference of ionic radii, or the expected corrugation of the short-ranged electrostatic force, or the potential corrugation found in atom-scattering experiments [16]. Therefore, the distortion of surface ions by the force of the tip has to be considered as a generally important contribution to the atomic corrugation [4]. As a consequence, the atomic corrugation found on terraces will always be significantly smaller than on the steps separating them.

It is interesting to note that the growth of $\mathrm{NaCl}$ films on different metal substrates exhibits significant differences. Foelsch et al. have studied the growth of $\mathrm{NaCl}$ on differently 
reconstructed copper surfaces [17]. They concluded that a registry of the $\mathrm{NaCl}$ films with charge modulations present on certain reconstructions strongly enhances the film-substrate interaction. The interaction can be strong enough to induce a facetting of the copper surface, where (111) facets are actually free of $\mathrm{NaCl}$. Growing $\mathrm{NaCl}$ on $\mathrm{Ge}(100)$ even results in a one-dimensional modulation of films up to a thickness of 15 monolayer [18]. The weak binding of $\mathrm{NaCl}$ to the $\mathrm{Cu}(111)$ surface may explain our finding that $\mathrm{NaCl}$ covers $\mathrm{Cu}(111)$ in micrometer-sized single-domain islands, overgrowing tens of substrate steps [12]. It would be worth a theoretical study to compare with the contrasting mechanism of the growth of well-ordered nanometer-sized $\mathrm{NaCl}$ islands on $\mathrm{Al}(111)$.

\section{3}

\section{Radiation damage in $\mathrm{KBr}$}

The irradiation of alkali halide crystals with ionizing radiation like low-energy electrons or ultraviolet light starts processes of defect creation and diffusion which strongly affect the crystal surface. Defect aggregation and desorption processes create surface features which often resem-
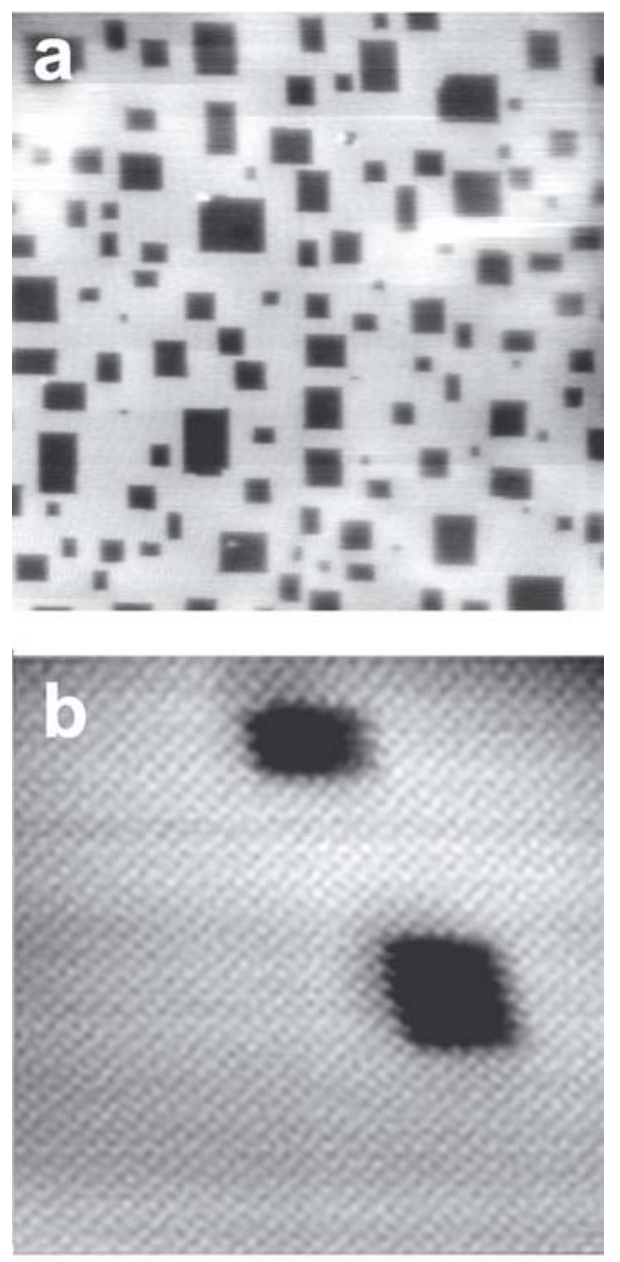

FIGURE 3 Topography of $\operatorname{KBr}(100)$ cleavage faces after irradiation with low-energy electrons. a frame size $100 \mathrm{~nm}$ b frame size $12.7 \mathrm{~nm}$. This frame was processed with a low-pass filter to enhance the atomic contrast on the surface. The pits have a size of 34 and 54 missing $\mathrm{KBr}$ molecules respectively. For details of the imaging conditions see [19] ble the symmetry of the respective crystal structure, pointing once more to the particular role of low-coordinated sites in all surface processes. Force microscopy is the only tool to image such nanometer-sized surface features and, thereby, contributes to the understanding of the underlying microscopic processes.

In a pioneering experiment, Wilson and Williams studied the evolution of KI surfaces exposed to ultraviolet light by scanning force microscopy in a dry nitrogen atmosphere. They found rectangular pits and hills with typical side lengths of $20-50 \mathrm{~nm}$ which were oriented along the main crystallographic direction [20]. Bennewitz et al. found triangular metallic islands on a $\mathrm{CaF}_{2}(111)$ surface after electron irradiation [21]. The development of rectangular pits after electron irradiation on the $\mathrm{KBr}(100)$ surface was described by Such et al. [22]. High-resolution force microscopy images of such pits are shown in Fig. 3. The electron dose applied in this experiment led to the desorption of a fraction of a monolayer in form of perfectly rectangular pits of one monolayer depth. Atomic resolution images proof that there are nearly no kinks in the steps surrounding the pits. The enhanced appearance of step atoms simplifies the determination of the atomically exact size of the pits. Furthermore, judging from the atomic contrast at steps and in the pits, we can assume that the stoichiometry of the surface is retained [19]. Combining this information with the finding of a correlation between desorption yield and step density on the surface, Such et al. suggested a complete picture of the desorption process based on halogen interstitial formation and predominant desorption from kink sites [23].

\section{4}

\section{Damping in dynamic force microscopy}

The damping of the tip oscillation due to tipsample interaction is an important aspect of dynamic force microscopy which has attracted much interest, since it may open a path to study dissipative mechanisms with atomic resolution. Basically, any dissipative interaction must reduce the energy of the tip oscillation. In our experiments, the amplitude is held constant, and a damping of the oscillation is recorded as increase of the excitation amplitude necessary to maintain the amplitude [24].

The damping is recorded simultaneously with the topography. Indeed, a strong variation of the damping is found on the atomic scale. One example is given in Fig. 4a. First of all, a strong contrast between the $\mathrm{Cu}$ substrate and the $\mathrm{NaCl}$ film partially covering the substrate is found. The atomic structure of the $\mathrm{NaCl}$ film is revealed, whereby step atoms cause significantly stronger damping. Obviously, the lower coordination plays an even more important role in the damping process than in the topographic imaging (compare Sect. 2). The interpretation of these damping measurements is controversial. The contrast between the $\mathrm{Cu}$ substrate and the $\mathrm{NaCl}$ film can be explained in terms of the work function difference between the two. The varying electrostatic force may cause a variation of Joule's dissipation in the semiconducting tip. Joule's dissipation as a source of damping in force microscopy has been demonstrated for heterogenous semiconductors [25]. For the atomic contrast, the situation is more complicated and several possible artifacts have been described [26]. The damping signal seems to depend on the atomic structure of the tip apex 

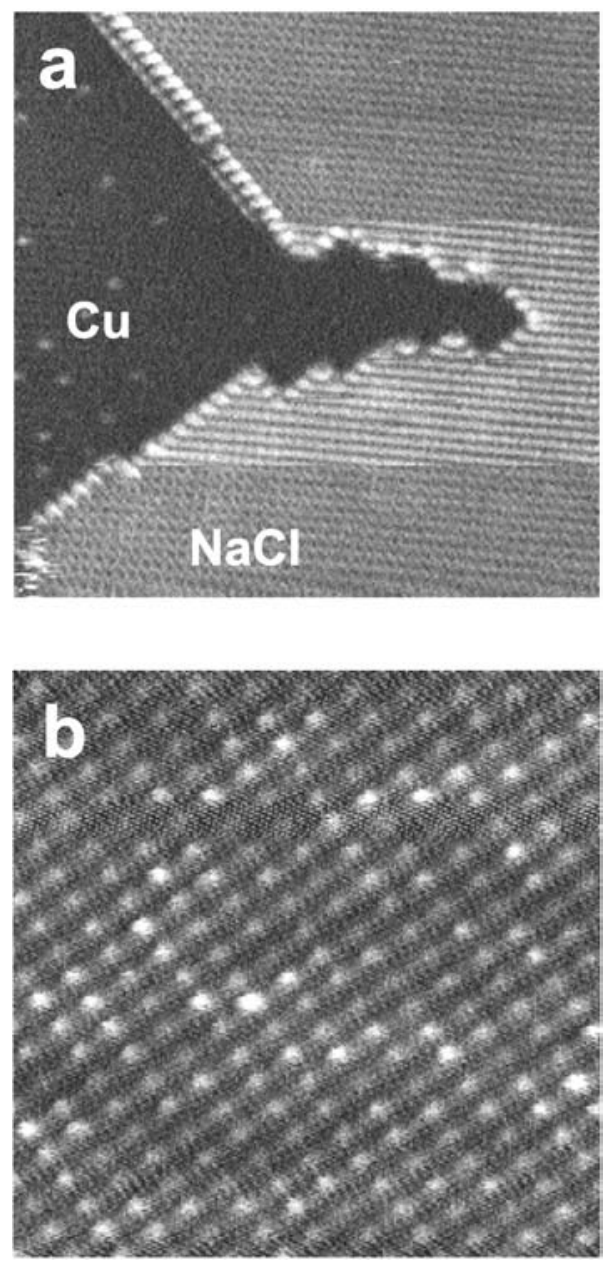

FIGURE 4 Maps of the damping of tip oscillation simultaneously recorded with topography maps (frame size $18 \mathrm{~nm}$ ). a $\mathrm{NaCl}$ film grown on $\mathrm{Cu}(111)$. Some sulphur impurities can be recognized on the $\mathrm{Cu}$ substrate. Note the enhanced damping at low-coordinated sites. For details of the imaging conditions see [14] b Cleavage face of a $\mathrm{KCl}_{0.6} \mathrm{Br}_{0.4}(100)$ crystal. The strong damping found on single atoms demonstrates the ability of dynamic force microscopy to distinguish different anions. For details of the imaging conditions see [4]

even more than the force signal [27]. Therefore, mechanisms of dissipation in a loosely bound tip structure have been proposed. However, the fascinating possibility of a microscopy of local phonon excitation is also under consideration [28]. For the moment, the damping maps are an experimental quantity that comes for free and provides often very helpful contrast between materials and at steps.

In an effort to understand the microscopic origins of the damping, we have studied mixed alkali halide crystals. The arbitrary distribution of ions with two different masses indeed provides an atomic contrast in the damping signal on a $\mathrm{KCl}_{0.6} \mathrm{Br}_{0.4}(100)$ surface. A clearly enhanced damping of the tip oscillation can be found above a number of protrusions in Fig. 4b. By comparing this number with the relative density of the anions, we can assign the enhanced damping to $\mathrm{Br}$ sites. A contrast between $\mathrm{Br}$ and $\mathrm{Cl}$ anions is also found in the the atomic corrugation of topography maps [4]. The atomic resolution of different chemical constituents is certainly an important step for the surface science of insulators. With respect to the current results, it is interesting to note that the random structure of ionic solid solutions [29] is preserved at the surface. However, the current results can not contribute to the understanding of dissipation phenomena on atomic scale. The topographic contrast on atomic scale can eventually create an artifact in the damping signal by convolution with long-range damping mechanisms. However, topography-independent atomic contrast in damping experiments has been demonstrated for the $\operatorname{Si}(111) 7 \times 7$ surface reconstruction [27]. For the results presented here, the damping signal can at least serve as high-contrast signal proving the ability of dynamic force microscopy to distinguish between different anions. The ultimate experiment proving sensitivity for atomic dissipation processes will probably have to deal with damping variations on different isotopes in a chemically homogenous crystal.

\section{Scratches in $\mathrm{KBr}$}

Force microscopy can also be used to study microscopic processes leading to wear. In a model experiment, we have scratched surfaces of $\mathrm{KBr}$ crystals by bringing the tip into contact with the surface, scanning forth and back, and increasing the normal load on the tip until some atomic
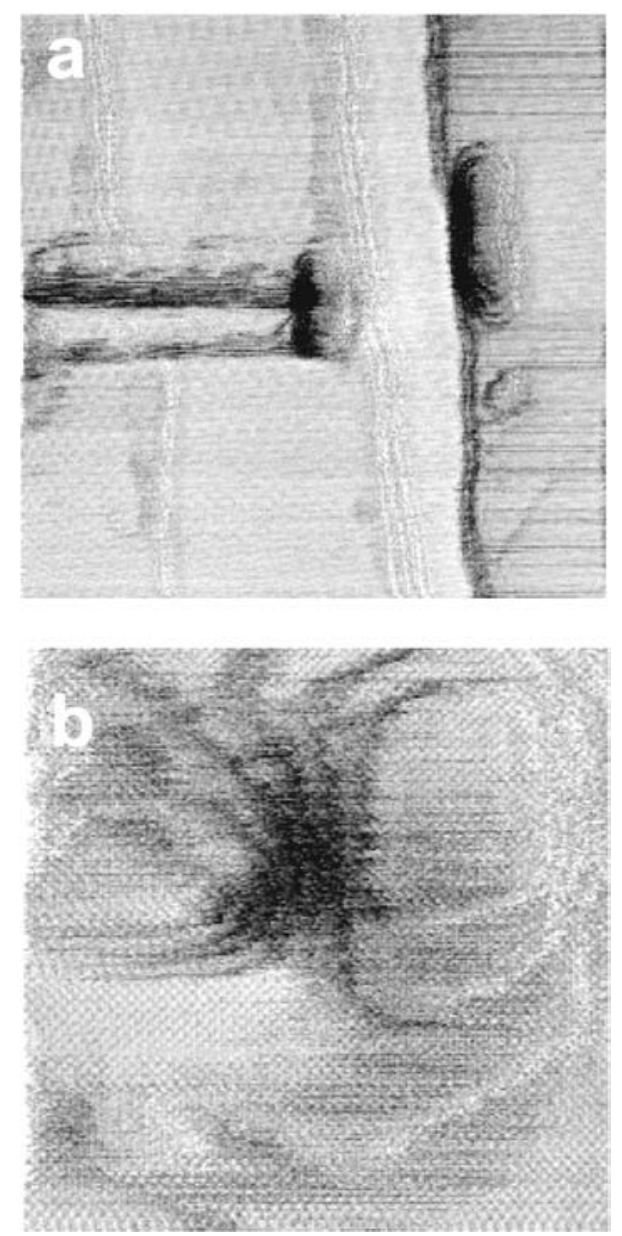

FIGURE 5 Lateral force maps acquired in contact mode around a scratch in a $\operatorname{KBr}(100)$ surface. a Overview showing one end of the scratch and the surrounding mounds. b Detail revealing the atomic structure of the terraces forming the mounds. For a detailed description of the experimental procedure see [30] 
layers had been removed from the tip (see Fig. 5a). In order to image the scratch and the debris around it, we reduced the load on the tip to the minimum and scanned the tip in contact, recording both the topography and the lateral force acting on the tip. The lateral force signal reveals the atomic periodicity of the surface via an atomic stick-slip process [31]. Figure 5b shows a typical result of such experiments [30]. The debris forms well organized terraces around the scratch, their atomic structure being in perfect registry with the crystal surface. Note that the atomic periodicity revealed in contact-mode force microscopy is not necessary the true atomic structure due to the finite size of the contact. However, the shift in registry between adjacent terraces can be resolved and, thereby, the height of the steps between the terraces proven to be monatomic. It is somewhat surprising and important for the further description of wear processes that the debris does not form an amorphous, irregular mound as might be inferred from macroscopic planing process. It can be assumed that the action of the tip releases and transports single molecules or very small clusters of $\mathrm{KBr}$, which reorganize in the form of smooth terraces like the islands seen in Sect. 2. Possibly, this re-crystallization of the wornoff material is also supported by the pressure of the tip in contact.

\section{6}

\section{Summary}

Force microscopy has been developed into a method providing atomic resolution on surfaces of alkali halides. The results contribute to several aspects of surface science including film growth, enhanced interaction at low-coordinated sites, radiation damage at the surface, or atomic scale wear. An interesting but not fully understood extension of the method may provide insight in dissipation processes at the atomic scale.

\section{REFERENCES}

1 S. Morita, R. Wiesendanger, E. Meyer: Noncontact Atomic Force Microscopy, NanoScience And Technology (Springer, Berlin, Germany, 2002)

2 R. Bennewitz, M. Bammerlin, E. Meyer: In: Noncontact Atomic Force Microscopy, NanoScience And Technology, ed. by S. Morita, R. Wiesendanger, E. Meyer (Springer, Berlin, 2002), pp. 93-108

3 F. Giessibl, H. Bielefeldt: Phys. Rev. B 61, 9968 (2000)
4 R. Bennewitz, O. Pfeiffer, S. Schär, V. Barwich, E. Meyer: Appl. Surf. Sci. 188, 232 (2002)

5 G. Bassett: Philos. Mag. 3, 1042 (1958)

6 H. Bethge: Surf. Sci. 3, 33 (1964)

7 N. Itoh, A. Stoneham: Materials modification by electronic excitation (Cambridge University Press, Cambridge, 2000)

8 C. Schwennicke, J. Schimmelpfennig, H. Pfnür: Surf. Sci. 293, 57 (1993)

9 K. Gloeckler, M. Sokolowski, A. Soukopp, E. Umbach: Phys. Rev. B 54, 7705 (1996)

10 R. Bennewitz, V. Barwich, M. Bammerlin, M. Guggisberg, C. Loppacher, A. Baratoff, E. Meyer, H.-J. Güntherodt: Surf. Sci. 438, 289 (1999)

11 W. Hebenstreit, J. Redinger, Z. Horozova, M. Schmid, R. Podloucky, P. Varga: Surf. Sci. 424, L321 (1999)

12 R. Bennewitz, M. Bammerlin, M. Guggisberg, C. Loppacher, A. Baratoff, E. Meyer, H.-J. Güntherodt: Surf. Interface Anal. 27, 462 (1999)

13 J. Repp, S. Fölsch, G. Meyer, K.-H. Rieder: Phys. Rev. Lett. 86, 252 (2001)

14 R. Bennewitz, A. Foster, L. Kantorovich, M. Bammerlin, C. Loppacher, S. Schär, M. Guggisberg, E. Meyer, H.-J. Güntherodt, A. Shluger: Phys. Rev. B 62, 2074 (2000)

15 Z. Gai, G. Farnan, J. Pierce, J. Shen: Appl. Phys. Lett. 81, 742 (2002)

16 G. Benedek, G. Brusdeylins, R. Bruce, J. Skofronick, J. Toennies: Phys. Rev. B 28, 2104 (1983)

17 S. Fölsch, A. Riemann, J. Repp, G. Meyer, and K.-H. Rieder: Phys. Rev. B 66, 161409 (2002)

18 W. Ernst, M. Eichmann, H. Pfnür, K.-L. Jonas, V. von Oeynhausen, K. Meiwes-Broer: Appl. Phys. Lett. 80, 2595 (2002)

19 R. Bennewitz, S. Schär, V. Barwich, O. Pfeiffer, E. Meyer, F. Krok, B. Such, J. Kolodzej, M. Szymonski: Surf. Sci. 474, L197 (2001)

20 R. Wilson, R. Williams: Nucl. Instrum. Meth. Phys. B 101, 122 (1995)

21 R. Bennewitz, D. Smith, M. Reichling: Phys. Rev. B 59, 8237 (1999)

22 B. Such, P. Czuba, P. Piatkowski, M. Szymonski: Surf. Sci. 451, 203 (2000)

23 B. Such, J. Kolodziej, P. Czuba, P. Piatkowski, P. Struski, F. Krok, M. Szymonski: Phys. Rev. Lett. 85, 2621 (2000)

24 C. Loppacher, R. Bennewitz, O. Pfeiffer, M. Guggisberg, M. Bammerlin, S. Schär, V. Barwich, A. Baratoff, E. Meyer: Phys. Rev. B 62, 13674 (2000)

25 T. Stowe, T. Kenny, D. Thomson, D. Rugar: Appl. Phys. Lett. 75, 2785 (1999)

26 H. Hug, A. Baratoff: In: Noncontact Atomic Force Microscopy, NanoScience And Technology, ed. by S. Morita, R. Wiesendanger, E. Meyer (Springer, Berlin, 2002), pp. 395-432

27 C. Loppacher, M. Bammerlin, M. Guggisberg, S. Schär, R. Bennewitz, A. Baratoff, E. Meyer, and H.-J. Güntherodt: Phys. Rev. B 62, 16944 (2000)

28 M.-Y. Mo, L. Kantorovich: J. Phys. C: Solid State Phys. 13, 1439 (2001)

29 U. Tinivella, M. Peressi, A. Baldereschi: J. Phys.: Condens. Matter 9, $11141(1997)$

30 E. Gnecco, R. Bennewitz, E. Meyer: Phys. Rev. Lett. 88, 215501 (2002)

31 R. Bennewitz, E. Gnecco, T. Gyalog, E. Meyer: Tribology Letters 10, 51 (2001) 